\title{
Mid-Infrared InAs/InAsSb Superlattice nBn Photodetector Monolithically Integrated onto Silicon
}

\author{
Evangelia Delli, ${ }^{*}{ }^{\dagger}$ Veronica Letka, ${ }^{\ddagger}$ Peter D. Hodgson, ${ }^{\ddagger}$ Eva Repiso, Jonathan P. Hayton,
} Adam P. Craig, Qi Lu, Richard Beanland, ${ }^{\ddagger}$ Anthony Krier, ${ }^{\ddagger}$ Andrew R. J. Marshall, and Peter J. Carrington* $* \dagger$

${ }^{\dagger}$ Engineering Department, Lancaster University, Bailrigg, Lancaster, LA1 4YW, United Kingdom

${ }^{\ddagger}$ Physics Department, Lancaster University, Bailrigg, Lancaster, LA1 4YB, United Kingdom

${ }^{\S}$ Physics Department, University of Warwick, Coventry, CA4 7AL, United Kingdom

\section{Supporting Information}

ABSTRACT: Mid-infrared (MIR) silicon photonics holds the potential for realizing next generation ultracompact spectroscopic systems for applications in gas sensing, defense, and medical diagnostics. The direct epitaxial growth of antimonidebased compound semiconductors on silicon provides a promising approach for extending the wavelength of silicon photonics to the longer infrared range. This paper reports on the fabrication of a high performance MIR photodetector directly grown onto silicon by molecular beam epitaxy. The device exhibited an extended cutoff wavelength at $\sim 5.5 \mu \mathrm{m}$ and a dark current density of $1.4 \times 10^{-2} \mathrm{~A} / \mathrm{cm}^{2}$ under $100 \mathrm{mV}$
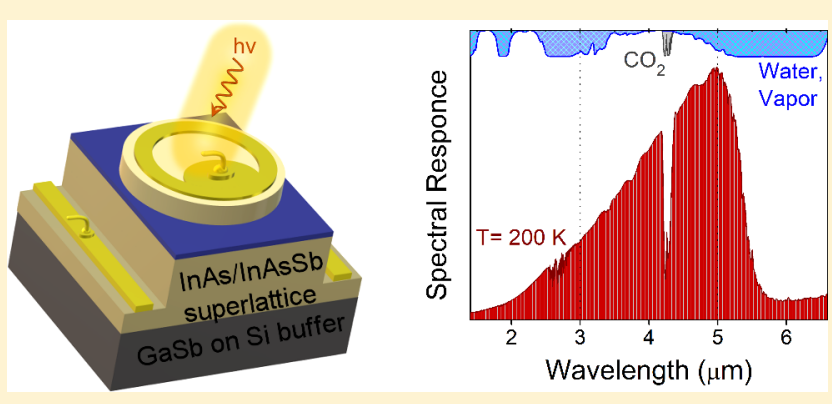
reverse bias at $200 \mathrm{~K}$. A responsivity of $0.88 \mathrm{~A} / \mathrm{W}$ and a specific detectivity in the order of $1.5 \times 10^{10}$ Jones was measured at 200 $\mathrm{K}$ under $100 \mathrm{mV}$ reverse bias operation. These results were achieved through the development of an innovative structure which incorporates a type-II InAs/InAsSb superlattice-based barrier $\mathrm{nBn}$ photodetector grown on a GaSb-on-silicon buffer layer. The difficulties in growing $\mathrm{GaSb}$ directly on silicon were overcome using a novel growth procedure consisting of an efficient AlSb interfacial misfit array, a two-step growth temperature procedure and dislocation filters resulting in a low defect density, antiphase domain free GaSb epitaxial layer on silicon. This work demonstrates that complex superlattice-based MIR photodetectors can be directly integrated onto a Si platform, which provides a pathway toward the realization of new, high performance, large area focal plane arrays and mid-infrared integrated photonic circuits.

KEYWORDS: type-II superlattice, photodetector, mid-infrared, molecular beam epitaxy, silicon photonics

$\mathrm{M}$ id-infrared (MIR) photodetectors operating between 2 and $8 \mu \mathrm{m}$ are of considerable technological importance for a wide range of applications including medical diagnostics, environmental monitoring, infrared imaging, and chemical sensing. Presently, commercially available sensors and focal plane arrays (FPAs) are predominantly based on mercurycadmium-telluride (MCT) materials. However, these devices require cooling to cryogenic temperatures to avoid excessive dark currents and suffer from poor compositional uniformity resulting in low reproducibility yields and high fabrication costs. ${ }^{1}$ Type II InAs/InAs $s_{1-x} \mathrm{Sb}_{x}$ superlattices (SLs) have emerged as a viable technology to compete with MCT. ${ }^{2}$ The type-II band alignment in the system leads to spatial separation of electrons and holes, providing remarkable flexibility for band structure engineering which enables the bandgap to be tuned across a wide wavelength range from 3 to $11 \mu \mathrm{m}$. ${ }^{3}$ It is possible to engineer the bandstructure to suppress intersub-band transitions to reduce nonradiative Auger recombination. As shown by Aytac et al. ${ }^{4}$, the Auger coefficients measured from InAs/InAsSb SLs are substantially lower compared to MCT for bandgap energies above $200 \mathrm{meV}$. Also, compared to conventional InAs/GaSb type-II SLs, significantly longer minority carrier lifetimes have been observed due to the absence of $\mathrm{Ga}$, which is associated with the formation of native defects. $^{5}$

Recently, the introduction of unipolar barriers based on type-II SLs has dramatically changed the design of infrared photodetectors. ${ }^{6}$ These structures (often referred to as $\mathrm{nBn}$ ) employ a wide-bandgap barrier layer positioned between narrow bandgap absorption and contact layers which blocks the flow of majority carriers (electrons), but not that of minority carriers (holes). A small applied bias falls almost completely across the barrier separating the photogenerated carriers. This almost entirely eliminates the electric field in the narrow-gap material which greatly suppresses the junction related Shockley-Read-Hall (SRH) dark current, resulting in significantly higher operating temperatures compared to

Received: November 8, 2018

Published: January 16, 2019 
conventional p-i-n photodiodes. ${ }^{7,8}$ The reduction in SRH generation provided by the $\mathrm{nBn}$ design also means the structure is less sensitive to crystalline defects and hence is well-suited for growing on lattice mismatched substrates such as silicon ( $\mathrm{Si}$ ). Furthermore, the InAs/InAsSb SL is also remarkably defect tolerant since the energy levels from the defect states are above the conduction band rather than the bandgap, making it less sensitive to SRH recombination. ${ }^{9,10}$

Implementation of $\mathrm{Si}$ as the main platform for the epitaxial growth of infrared detectors is extremely attractive for the future development of focal plane arrays (FPAs). Conventionally, arrays of photodetectors are connected to a Si readout integrated circuit (ROIC) using hybrid flip-chip bonding. However, this fabrication process is expensive and consists of several steps including wafer bonding and substrate thinning, which limits the yield and array size, restricting their potential for mass production. ${ }^{11}$ Heteroepitaxial growth on Si offers the possibility for monolithic integration of FPAs and ROICs; this provides significant advantages due to the availability of low cost, mechanically robust and large area $\mathrm{Si}$ wafers, which are compatible to the ROIC, allowing fabrication of large FPA arrays with reliable long-term thermal cycling. Furthermore, heterogeneous integration of MIR photodetectors on $\mathrm{Si}$ is an essential step in the development of chip-scale and high volume production of MIR silicon photonic systems. ${ }^{12}$ Integration of MIR photodetector devices along with other active and passive components onto $\mathrm{Si}$ will enable complete on-chip integration of electronic and photonic systems, leading to a new generation of high performance MIR photonic devices.

This work reports on the heteroepitaxial growth of a type II $\mathrm{InAs} / \mathrm{InAsSb} \mathrm{SL} \mathrm{nBn}$ photodetector directly onto Si. High quality, antiphase domain-free $\mathrm{GaSb}$ epilayers on $\mathrm{Si}$ were realized using an advanced buffer layer design which utilizes a combination of an AlSb nucleation layer, a two-step $\mathrm{GaSb}$ growth temperature procedure and $\mathrm{AlSb} / \mathrm{GaSb}$ dislocation filters. The resulting photodetector exhibited an extended $50 \%$ cutoff wavelength close to $6 \mu \mathrm{m}$ at room temperature, and $25.6 \%$ external quantum efficiency at $200 \mathrm{~K}$, without an antireflection coating. The device demonstrated a midwavelength, $100 \mathrm{mV}$ reverse bias specific detectivity in the order of $10^{10}$ Jones at $200 \mathrm{~K}$.

\section{RESULTS AND DISCUSSION}

Design and Analysis of InAs/InAsSb SL nBn Structure. Samples were grown on $\mathrm{Si}(100)$ substrates with a $4^{\circ}$ offcut toward $[0-11]$. The GaSb buffer layer was first grown using a thin 17 monolayer (ML) AlSb nucleation layer. ${ }^{13}$ This improves the quality of bulk $\mathrm{GaSb}$ grown on $\mathrm{Si}$ through the formation of three-dimensional (3-D) islands on the surface, which reduces the diffusion length of $\mathrm{Ga}$ atoms, promoting planar growth of GaSb. Second, it is possible to relieve the strain at the Si:III-V interface via the formation of a periodic array of $90^{\circ}$ interfacial misfit dislocations (IMF), which propagate laterally rather than vertically to the horizontal plane. ${ }^{14}$ Figure la shows a false-color transmission electron microscopy (TEM) image of the AlSb islands created at the Si:III-V interface, which demonstrated a hemispherical, noncoalesced shape with an average height of $8.9 \mathrm{~nm}$. The high magnification image of Figure $1 \mathrm{~b}$ shows the IMF dislocation array created at the interface, where a Fourier filter was applied to extract the atomic arrangement. The average dislocations spacing is approximately $3.35 \mathrm{~nm}$, very
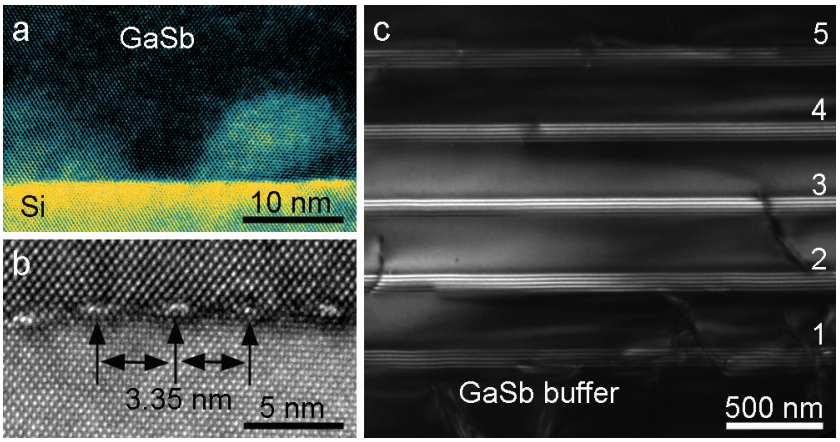

Figure 1. (a) False-color high resolution TEM image taken along the $\mathrm{III}-\mathrm{V} / \mathrm{Si}$ interface, showing the formation of AlSb islands. (b) High resolution image was taken along the [110] showing the misfit dislocations with spacing of $3.35 \mathrm{~nm}$. (c) Cross-sectional dark field [220] TEM micrograph of the area with the $\mathrm{AlSb} / \mathrm{GaSb}$ dislocation filter layers. The numbers indicate the five sets of DFSLs.

close to the $3.34 \mathrm{~nm}$ theoretical spacing value ${ }^{15}$ of IMFs for complete misfit strain relaxation, indicating a periodic formation every eight atomic planes. Following deposition of the AlSb, a two-step growth temperature method was employed for the epitaxial growth of a $2 \mu \mathrm{m}$ thick $\mathrm{GaSb}$ buffer layer (further details are given in the Materials and Methods and Supporting Information). The majority of threading dislocations (TDs) originating from the Si:III-V interface were well confined within the first $700 \mathrm{~nm}$ of the $\mathrm{GaSb}$ buffer which was also antiphase domains (APD) free. To improve the material quality further, five sets of strained $\mathrm{AlSb} /$ $\mathrm{GaSb}$ dislocation-filter superlattices (DFSLs) were grown on top of the two-step GaSb buffer layer. Dislocation filters encourage bending of TDs by introducing additional strain which promotes their lateral propagation and increases the likelihood that they will join with one another and annihilate. ${ }^{16}$ Each set of DFSL is made up of five iterations of $10 \mathrm{~nm} \mathrm{GaSb} /$ $10 \mathrm{~nm} \mathrm{AlSb}$, with $300 \mathrm{~nm}$ of GaSb spacer layer between the sets. Figure 1c shows the cross-sectional TEM image of the area with the dislocation filters. The filters significantly promoted the annihilation of TDs, either by blocking their vertical propagation or forcing them to move parallel to the growth surface. The DFSLs significantly reduced the surface TDs density down to $3 \times 10^{7} \mathrm{~cm}^{-2}$.

The detector was grown on top of the buffer layer which consists of an absorption layer superlattice (ALSL) and a contact layer superlattice (CLSL), both of which are made up of $\mathrm{n}$-doped InAs/InAsSb layers, as shown in Figure 2a. The total thickness of these structures is $4 \mu \mathrm{m}$ and $400 \mathrm{~nm}$ respectively, with a thin $100 \mathrm{~nm}$ AlGaAsSb electron blocking barrier layer separating them. The AlGaAsSb barrier layer provides a high conduction band offset and a small valence band offset of a few tens of $\mathrm{meV}$, enabling the unimpeded flow of the photogenerated minority carriers (holes), as shown in Figure $2 \mathrm{~b}$. Figure $2 \mathrm{c}$ shows the bandstructure of the InAs/ InAsSb SL along with the first electron/hole energy levels and wave functions $\left(\Psi_{\mathrm{i}}^{2}\right)$ calculated using Nextnano (details about the Nextnano simulation can be found in the Supporting Information). The bandgap of the SL and hole localization energy mainly depends on the $\mathrm{Sb}$ composition and the thickness of the InAsSb layer, which introduces compressive strain. ${ }^{17}$ This must be compensated by the tensile strain in the InAs layer, which determines the electron energy. All parameters need to be simultaneously optimized to achieve 
a

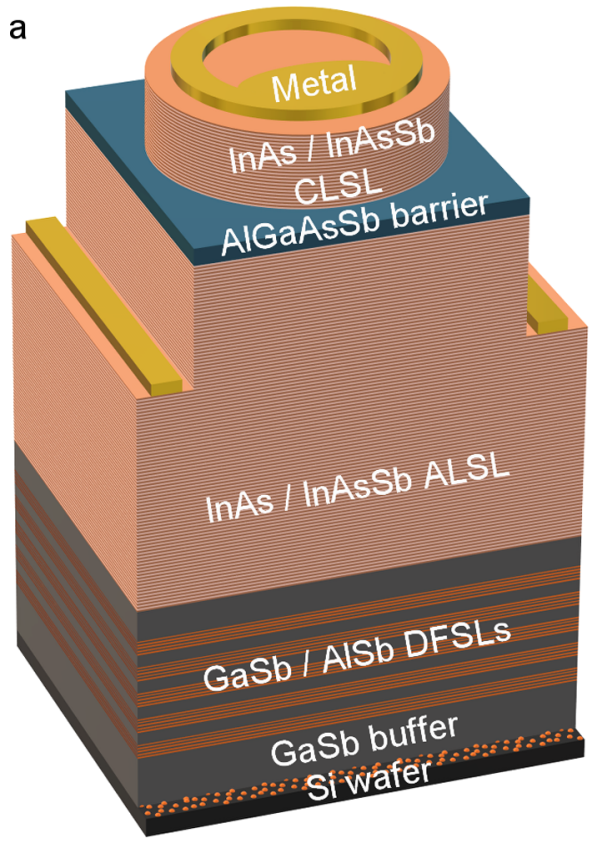

b
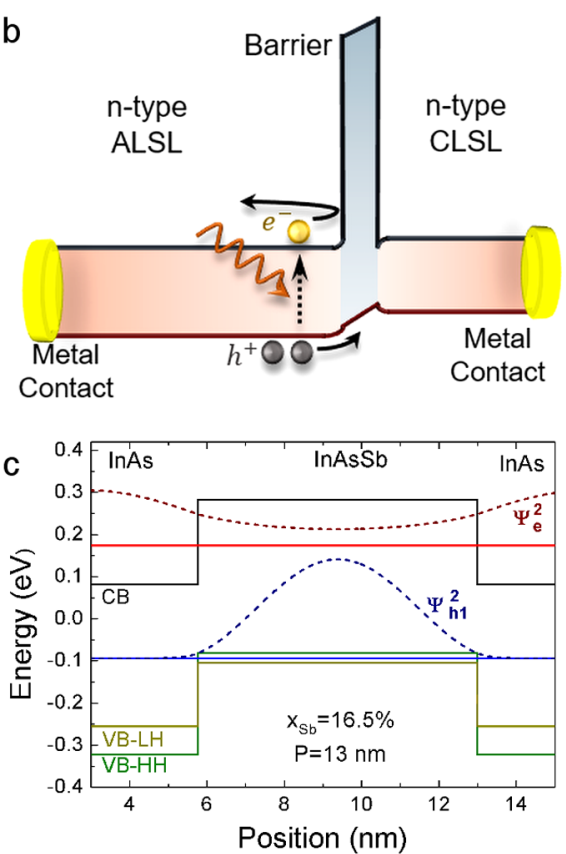
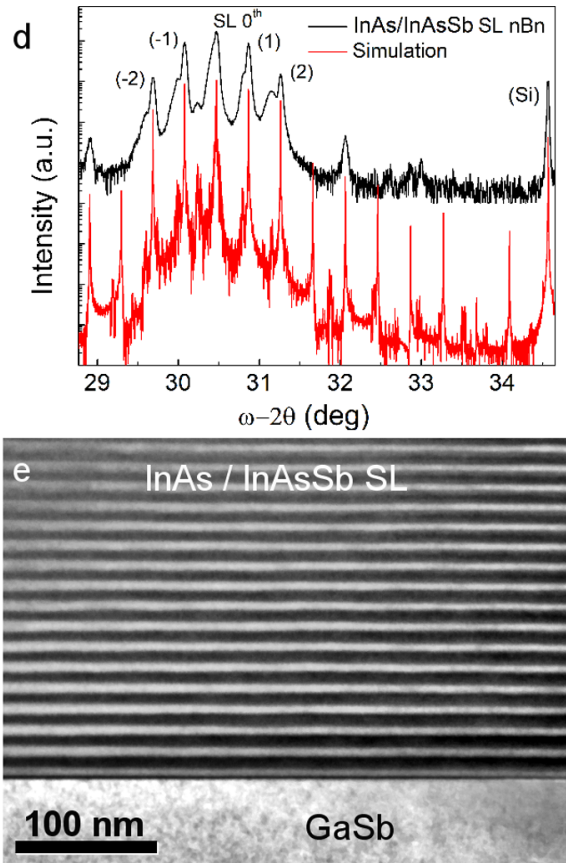

Figure 2. (a) Schematic of the InAs/InAsSb type-II SL nBn structure grown on top of the GaSb/AlSb/Si buffer, using AlSb/GaSb dislocation filters. (b) Diagram of the full device's bandgap structure showing the barrier layer which blocks the flow of electrons. (c) Calculated InAs/InAsSb SL bandstructure with first electron and hole energy levels and wave functions $\left(\Psi_{\mathrm{i}}^{2}\right)$. (d) Experimental and simulated high resolution X-ray diffraction pattern of the SL. (e) High resolution cross section TEM image of the InAs/InAsSb SL epilayers.

strain balancing ${ }^{18}$ and to tune the SL bandgap energy over a specific wavelength range. A strain-balanced InAs/InAsSb SL was designed (see Supporting Information) to cover the 3-5 $\mu \mathrm{m}$ atmospheric window, with an $\mathrm{Sb}$ content of $16 \%$ and a period of $13.3 \mathrm{~nm}(\operatorname{InAs}=5.9 \mathrm{~nm}, \operatorname{InAsSb}=7.4 \mathrm{~nm})$. The wave function overlap was calculated to be approximately $55 \%$.

The $\omega$ - $2 \theta$ high resolution X-ray diffraction (XRD) pattern of the InAs/InAsSb $\mathrm{nBn}$ detector is shown in Figure 2d. Many intense and well-defined satellite peaks can be observed, indicating excellent crystalline quality. Simulation of the SL $\mathrm{XRD}$ pattern indicates a SL period thickness of $13 \mathrm{~nm}$ (InAs = $5.8 \mathrm{~nm}, \mathrm{InAsSb}=7.2 \mathrm{~nm}$ ), with an Sb content of $16.5 \%$ in the InAsSb layer, which closely match the target SL values. The high-resolution TEM of the SL $\mathrm{nBn}$ active region is presented in Figure 2e showing abrupt interfaces with low $\mathrm{Sb}$ segregation. The SL displayed a planar growth mode without any characteristic wavy deformation behavior as observed recently for similar Sb-based multilayer structures integrated on $\mathrm{Si}^{19}$

Photoluminescence (PL) measurements were carried out at $5 \mathrm{~K}$ and revealed a strong SL emission peak at $4.7 \mu \mathrm{m}$, as shown in Figure 3a. This corresponds to a carrier recombination energy of $264 \mathrm{meV}$, which is in excellent agreement with the $268 \mathrm{meV}$ ground-state recombination energy calculated using Nextnano. Figure $3 \mathrm{~b}$ shows the integrated PL intensity versus laser power, which was fitted using the equation $I=\alpha P^{\beta}$, where $I$ is the PL integrated intensity, $P$ is the laser excitation power, $\alpha$ is a constant, and $\beta$ is related to the recombination processes. ${ }^{20} \mathrm{~A}$ gradient, $\beta$, of $1.08 \pm 0.03$ was found, indicating radiative recombination is the dominant recombination process, despite the remaining presence of threading dislocations $\left(3 \times 10^{7} \mathrm{~cm}^{-2}\right)$ inside the active region.

Electrical Characterization. The as-grown type-II SL nBn on $\mathrm{Si}$ sample was processed into nonisolated circular-mesa photodetectors using standard photolithography and wet- chemical etching. Figure $4 \mathrm{a}$ shows the dark current $J_{\text {dark }}$ characteristics of the $\mathrm{nBn}$ detector with a mesa diameter of $50 \mu \mathrm{m}$ as a function of bias voltage and temperature. The detector's dark current density increased by 5 orders of magnitude with increasing temperature, as shown in Figure 4b. At a reverse bias voltage of $100 \mathrm{mV}$ the room temperature dark current density was $8.65 \times 10^{-1} \mathrm{Acm}^{-2}$, and decreased to $1.2 \times$ $10^{-3} \mathrm{Acm}^{-2}$ at $160 \mathrm{~K}$. These results are lower compared to previous reports of Sb-based MIR detector devices integrated onto $\mathrm{Si}^{21,22}$ However, the dark current values are still higher compared to that exhibited by $\mathrm{HgCdTe}$ photodiodes which can be described by Rule $07 .{ }^{23}$ For a $50 \%$ cutoff wavelength close to $5.2 \mu \mathrm{m}$ at $160 \mathrm{~K}$ Rule 07 predicts that the dark current should be about 2 orders of magnitude lower to the value reported here.

The dark current generation mechanism of the device was investigated using the Arrhenius plot of dark current density data with $V_{\mathrm{b}}=-100 \mathrm{mV}$ as shown in Figure $4 \mathrm{~b}$. The equation $J_{\text {dark }} \approx T^{3 / 2} \exp \left(-E_{\mathrm{a}} / k T\right)$ was used to fit the data, where $E_{\mathrm{a}}$ is the activation energy and $\mathrm{k}$ is the Boltzmann constant. ${ }^{24}$ The activation energy was found to be $173 \mathrm{meV}$, which is slightly higher than half the theoretically predicted $268 \mathrm{meV}$ groundstate transition energy at low temperature. Therefore, this value indicates that for temperature above $110 \mathrm{~K}$ the dark current is dominated by generation-recombination processes, with an additional diffusion contribution, which explains why the dark current density is much higher compared to the theoretical value predicted by Rule 07 . For temperatures below $100 \mathrm{~K}$ the current can be ascribed to the noise floor of the experimental setup. The dynamic resistance-area product $\left(R_{\mathrm{d}} A\right)$ of the device at various temperatures shown in Figure $4 \mathrm{~b}$ exhibits inverse behavior compared to the dark current density, decreasing from $77.7 \times 10^{2} \Omega \mathrm{cm}^{2}$ at $110 \mathrm{~K}$ to $10.6 \Omega$ $\mathrm{cm}^{2}$ at $200 \mathrm{~K}$. 
a

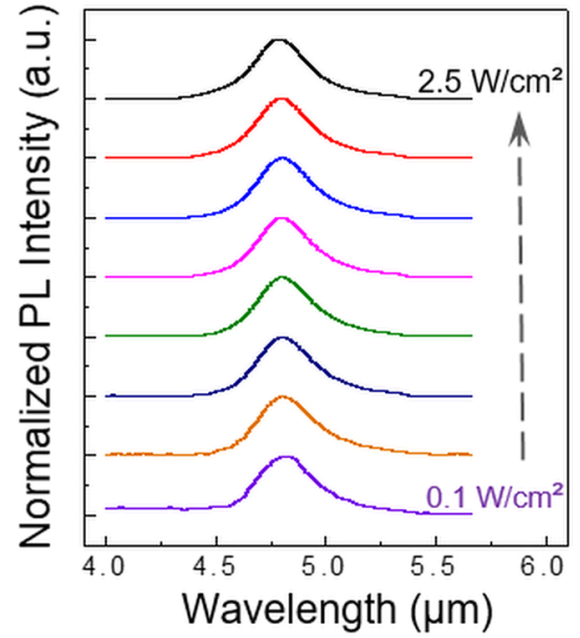

b

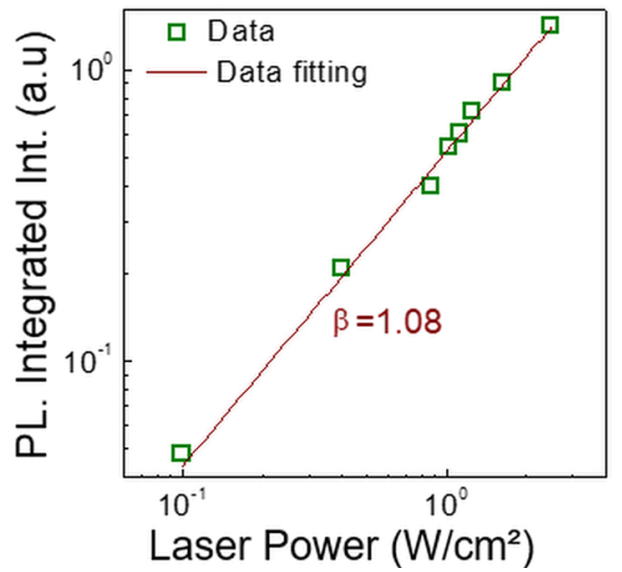

Figure 3. (a) Normalized PL intensity signal of the unprocessed material obtained at $5 \mathrm{~K}$ for different laser excitation powers. (b) Integrated PL peak intensity dependence on the laser excitation power at $5 \mathrm{~K}$.

Optical Properties. To investigate the optoelectronic performance of the photodetector, the device was loaded into a controllable low-temperature cryostat to perform temperature dependent optical characterization (see Supporting Information). Figure 5a shows the normalized spectral response of the detector obtained at three different temperatures under reverse bias operation of $V_{\mathrm{b}}=100 \mathrm{mV}$. The photoresponse covered a wide spectral range from 2.5 up to $6 \mu \mathrm{m}$, with a $50 \%$ cutoff wavelength, which varied relatively weakly over a wide range of temperatures: at $200 \mathrm{~K}$ the device exhibited a $50 \%$ cutoff at $5.40 \mu \mathrm{m}$, which moved to $5.86 \mu \mathrm{m}$ at $300 \mathrm{~K}$. The device responsivity was subsequently measured for temperatures ranging from 160 to $220 \mathrm{~K}$ in the spectral range 3.7 to 4.9 $\mu \mathrm{m}$ using a $823 \mathrm{~K}$ blackbody emitter under top-side illumination. Figure $5 \mathrm{~b}$ shows the responsivity under -100 $\mathrm{mV}$ bias operation, which exhibits an increasing trend up to a maximum responsivity of $0.88 \mathrm{~A} / \mathrm{W}$ at $200 \mathrm{~K}$, resulting in an external efficiency of $25.6 \%$ for the spectral range 3.7 to 4.9 $\mu \mathrm{m}$. For temperatures higher than $200 \mathrm{~K}$ the responsivity decreased, dropping to $0.5 \mathrm{~A} / \mathrm{W}$ at $220 \mathrm{~K}$.

The shot/thermal noise limited specific detectivity, $D^{*}$, was calculated using the equation ${ }^{25}$
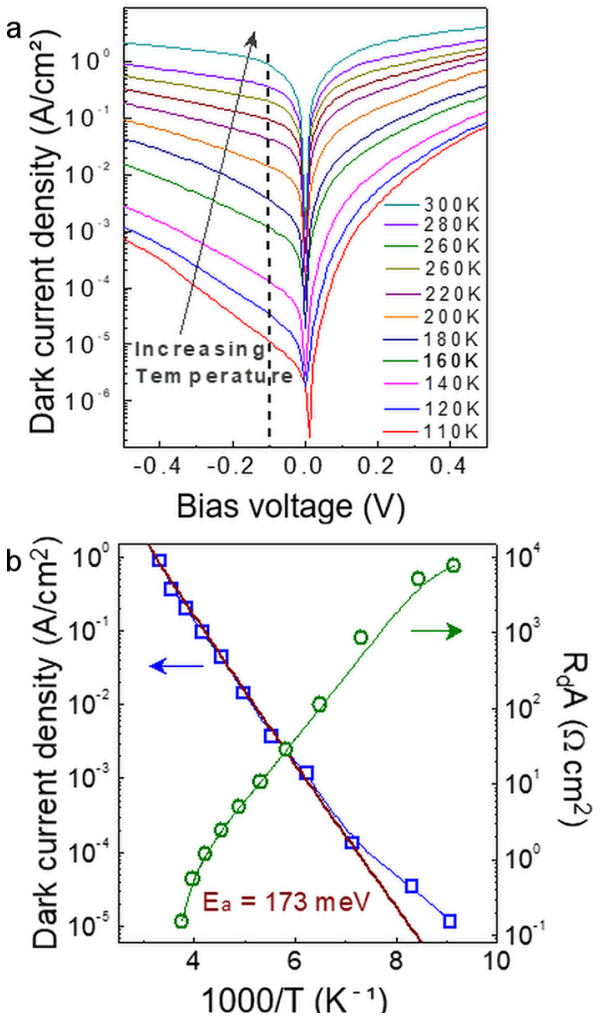

Figure 4. (a) Bias voltage dependence of the dark current density. The diameter of the circular device was $50 \mu \mathrm{m}$. The dashed line indicates the position of the $100 \mathrm{mV}$ reverse bias. (b) Dark currents and dynamic resistance-area product as a function of temperature with a reverse bias voltage of $V_{\mathrm{b}}=100 \mathrm{mV}$. The data was fit in the high temperature region using an activation energy of $173 \mathrm{meV}$.

$$
D^{*}=\frac{R}{\sqrt{\left(2 q J_{\mathrm{dark}}+\frac{4 k T}{R_{\mathrm{d}} A}\right)}}
$$

where $R$ is the total responsivity in A/W for a reverse operating bias voltage $100 \mathrm{mV}$ and $q$ is the elementary charge. The calculated detectivity was $3.65 \times 10^{10}$ Jones and $1.5 \times 10^{10}$ Jones at 160 and $200 \mathrm{~K}$, respectively, as shown in Figure 5b. These results are higher than bulk InSb detectors grown previously on $\mathrm{Si}^{26,27}$ and that of InAs/GaSb SLs grown on GaAs. ${ }^{28}$ This can be attributed to the improved structural quality of the $\mathrm{GaSb} / \mathrm{Si}$ buffer layers, and the reduced defect sensitivity provided by the InAs/InAsSb SL nBn design. However, they are about an order of magnitude lower than state-of-the-art InAs/InAsSb SL nBn photodetectors integrated on native $\mathrm{GaSb}$ substrate reported recently by Nasa $\mathrm{JPL}^{9}$ (a summary of recent results is given in the Supporting Information). For a more complete consideration, it should be pointed out that since the devices are topside illuminated, performance is limited by losses due to light absorption within the CLSL region and contact reflection. Therefore, higher $D^{*}$ values could be obtained using backside illumination and by applying a suitable antireflection coating on the detector. Finally, additional improvements can be expected by reducing the TD density to the level of $10^{-6} \mathrm{~cm}^{-2}$ or below through further optimization of the DFSLs which currently limits the G-R dark current. 

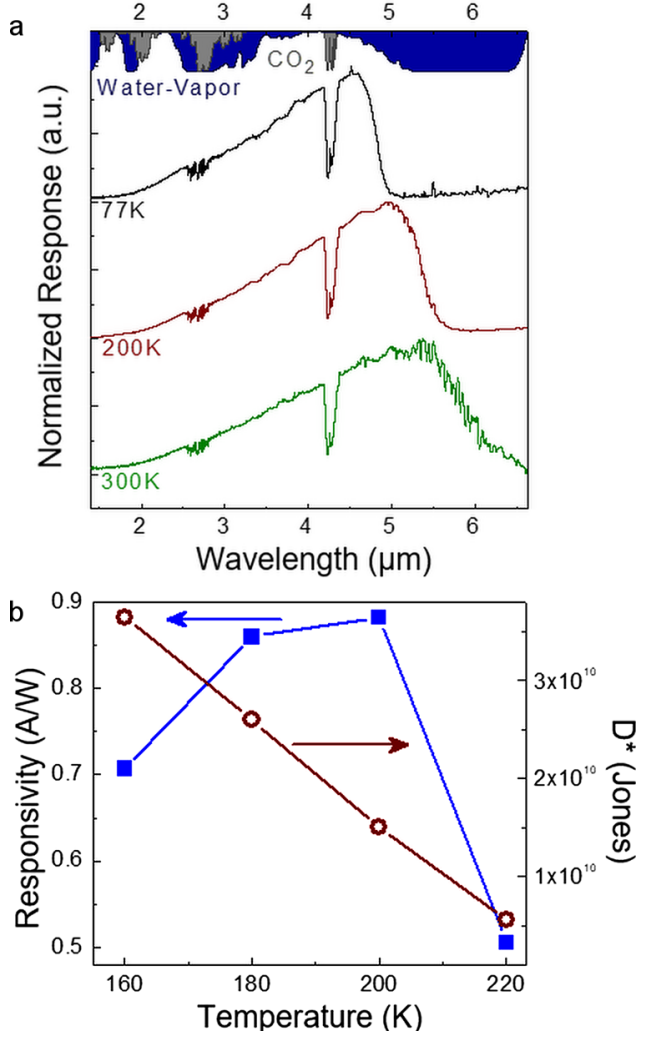

Figure 5. (a) Normalized spectral response curves for the detector at different temperatures $\left(\mathrm{CO}_{2}\right.$ absorption shown in gray, water vapor absorption in blue). (b) Total spectral responsivity and specific detectivity, $D^{*}$, values measured as a function of temperature in the 3.7 to $4.9 \mu \mathrm{m}$ spectral band. The operation bias voltage for all measurements was $100 \mathrm{mV}$.

\section{CONCLUSION}

In conclusion, a high-performance MIR $\mathrm{nBn}$ photodetector based on InAs/InAsSb type-II superlattice was heteroepitaxially integrated onto $\mathrm{Si}$ using a GaSb/Si buffer layer. The first part of the buffer layer was grown using an optimized IMF array and a two-step growth temperature $\mathrm{GaSb}$ layer, which confined most of the TDs within the first $700 \mathrm{~nm}$ of the buffer and exhibited no APDs. The second part consisted of five iterations of $\mathrm{AlSb} / \mathrm{GaSb}$ dislocation filter layers which successfully reduced the TD down to $3 \times 10^{7} \mathrm{~cm}^{-2}$. The photodetector exhibited an external quantum efficiency of $25.6 \%$ at $200 \mathrm{~K}$ and maximum specific detectivity of $3.65 \times$ $10^{10}$ Jones under topside illumination at $160 \mathrm{~K}$. Electrical characterization showed that the dark current generation was primarily dominated by generation-recombination current. These results will allow cost-effective silicon photonics and new thermoelectrically cooled detection systems on Si to be developed operating in the technologically important MIR spectral range from 3 to $5 \mu \mathrm{m}$, opening the way for a wide range of applications.

\section{MATERIALS AND METHODS}

Si Wafer Surface Preparation. The integration of high quality III-V semiconductor layers onto a silicon substrate requires a perfectly clean and plane growth surface, as any contaminants present could act as nucleation centers for TDs and drastically degrade the quality of the epilayer. Various exsitu surface cleaning techniques have been developed, requiring multiple steps of wet and/or dry etching. In this work, an in situ thermal cleaning technique was employed for the $\mathrm{Si}$ wafers. The thermal treatment consisted of several thermal cycles with the maximum temperature reaching 1000 ${ }^{\circ} \mathrm{C}$. Such high temperature is necessary to remove the surface oxide, simultaneously eliminating any carbon contaminations present on the surface.

MBE Growth of InAs/InAsSb SL nBn. The samples studied here were grown using a solid source Veeco GENxplor MBE system equipped with valved arsenic (As) and antimony (Sb) cracker cells. The structure was grown directly on an ntype $\mathrm{Si}$ substrate with $4^{\circ}$ miscut toward [0-11] direction. Following oxide desorption, the substrate was cooled to 490 ${ }^{\circ} \mathrm{C}$ and exposed to an $\mathrm{Sb}$ flux for $5 \mathrm{~min}$, followed by the deposition of a $17 \mathrm{ML}$ thick AlSb nucleation layer using a growth rate of $0.36 \mathrm{ML} / \mathrm{sec}$ at a growth temperature of $490{ }^{\circ} \mathrm{C}$. A $2 \mu \mathrm{m}$ thick $\mathrm{GaSb}$ buffer layer was grown using a two-step growth temperature technique with a Ga growth rate of 0.66 $\mathrm{ML} / \mathrm{sec}$. Initially a $1.5 \mu \mathrm{m}$ thick GaSb layer was grown at the same growth temperature as the AlSb layer $\left(490{ }^{\circ} \mathrm{C}\right)$ to maintain the shape of the AlSb islands and ensure planar $2 \mathrm{D}$ growth. Then, the temperature was increased to $515^{\circ} \mathrm{C}$, which is the optimum growth temperature for high crystalline quality $\mathrm{GaSb}$, and a further $500 \mathrm{~nm}$ of GaSb was deposited. Next, a series of AlSb $(10 \mathrm{~nm}) / \mathrm{GaSb}(10 \mathrm{~nm})$ strained-layer DFSLs were grown. The InAs/InAsSb type-II SL was grown at $430{ }^{\circ} \mathrm{C}$ and consisted of: a 320 period InAs/InAsSb ALSL, an $\mathrm{Al}_{0.9} \mathrm{Ga}_{0.1} \mathrm{As}_{0.1} \mathrm{Sb}_{0.9}$ electron blocking barrier layer and a 30 period InAs/InAsSb CLSL. An optimized shutter sequence was used at the SL interfaces consisting of $\mathrm{Sb} / \mathrm{As}$ soaks to create abrupt layers and reduce $\mathrm{Sb}$ segregation. The growth rates were $1 \mathrm{ML} / \mathrm{sec}$ for both InAsSb and InAs layers, and the intentional doping was Te: $5 \times 10^{16} \mathrm{~cm}^{-3}$ and $2 \times 10^{17} \mathrm{~cm}^{-3}$ for the ALSL and CLSL, respectively.

Device Fabrication. Standard photolithography and chemical etching techniques were employed to process the as-grown wafer into photodetector devices. The processing consists of two UV photolithography, two etching and two metal evaporation steps. The metallic contacts were formed using $\mathrm{Ti}(20 \mathrm{~nm}) / \mathrm{Au}(200 \mathrm{~nm})$. The mesa pattern etching was carried out using citric acid and $\mathrm{H}_{2} \mathrm{O}_{2}$ with a ratio of $2: 1$. A hydrochloric acid solution [HCL (1): $\left.\mathrm{H}_{2} \mathrm{O}_{2}(1): \mathrm{H}_{2} \mathrm{O}(5)\right]$ was used for the lower contact pattern etching process. No mechanical wafer thinning or $\mathrm{SiO}_{2}$ isolation processes were performed prior to the metal contact deposition.

Structure Characterization. Electron channeling contrast images (ECCI) were collected using a Zeiss Gemini SEM with a solid-state backscatter detector operating at $20 \mathrm{kV}$. TEM specimens were prepared using conventional routes, i.e grinding, polishing and ion milling to electron transparency using $6 \mathrm{kV} \mathrm{Ar}{ }^{+}$ions. A final low energy $(1.5 \mathrm{kV})$ ion mill was used to reduce surface damage. Samples were examined in JEOL 2100 and JEOL 2100 Plus TEMs operating at $200 \mathrm{kV}$. AFM surface characterization was performed using a Multimode Atomic Force Microscope in tapping mode, using a Nanoscope 8 controller. High resolution X-ray measurements were carried out using a Bede QC200 Diffractometer. Fitting of the X-rays pattern was performed using the corresponding RADS Mercury software.

Electrical Characterization. The electrical properties of the SL photodetectors were obtained using a Lakeshore TTPX low-temperature probe station, equipped with a radiation shield and Keithley source measurement unit and was 
controlled remotely with LabVIEW software. The processed chips were loaded into the probe station and then cooled for temperature dependent $I-V$ characterization.

Optical Characterization. The photoluminescence measurements were performed using a $785 \mathrm{~nm}$ diode laser with a beam diameter of about $1 \mathrm{~mm}$, which corresponds to a maximum laser excitation power density at the sample surface of approximately $2.5 \mathrm{~W} \mathrm{~cm}$. The as-grown wafer was mounted in a continuous flow $\mathrm{He}$ cryostat which allowed control of the sample temperature. A Bruker Vertex 70 Fourier Transform Infrared (FTIR) Spectrometer and a $77 \mathrm{~K} \mathrm{InSb}$ photodiode detector were used for signal detection in step scan mode. The input signal was processed by a Bruker digitizer and recorded using OPUS readout software. Photoresponse measurements were performed using an OptistatDN-V2 cryostat and an FTIR spectrometer. The processed chips were wire-bonded, mounted onto standard TO headers, loaded in the cryostat and then cooled to the temperature of interest. A Stanford Research Systems SR570 preamplifier provided the system with reverse operating bias voltage of $-100 \mathrm{mV}$. Finally, device responsivity was measured using a Landcal P550P blackbody emitter as a light source operating at $823 \mathrm{~K}$. The device was again mounted inside the Optistat DN-V2 cryostat, connected to the SR570 preamplifier, and biased at $-100 \mathrm{mV}$. A mechanical chopper operating at $<200 \mathrm{~Hz}$ was used to modulate the light emitted from the blackbody source. The photocurrent was measured using a SRS SR830 lock-in amplifier, which used the frequency of the chopper as a reference. A 3.7 to $4.9 \mu \mathrm{m}$ band-pass filter was used to isolate the MIR atmospheric absorption window and emulate typical commercial detector operating conditions. The overall external quantum efficiency for this band-pass was calculated and reported.

\section{ASSOCIATED CONTENT}

\section{S Supporting Information}

The Supporting Information is available free of charge on the ACS Publications website at DOI: 10.1021/acsphotonics.8b01550.

(1) TEM analysis of the GaSb/AlSb buffer; (2) Fourier filtering microstructure of the IMF array; (3) AFM characterization of the surface; (4) ECCI characterization of the surface; (5) Temperature dependent spectral response; (6) Simulation of the InAs/InAsSb type II; (7) Summary of recent results (PDF).

\section{AUTHOR INFORMATION}

\section{Corresponding Authors}

*E-mail: e.delli@lancaster.ac.uk.

*E-mail: p.carrington@lancaster.ac.uk.

\section{ORCID}

Peter J. Carrington: 0000-0003-2107-5602

\section{Author Contributions}

E.D., A.R.J.M., and P.J.C. proposed the idea and coordinated the project. E.D., P.D.H., and P.J.C. developed the growth of the $\mathrm{GaSb} / \mathrm{Si}$ buffer layers. V.L., J.P.H., A.P.C., and A.R.J.M. designed the growth of the $\mathrm{nBn}$ structure. E.D., E.R., and P.J.C. developed the Nextnano simulations. E.D. carried out XRD characterization and device processing. E.D. and P.D.H. performed the AFM measurements and analysis. R.B. performed the TEM and ECCI measurements. E.D. carried out photoluminescence measurements, with help from E.R.,
Q.L., and A.K. The detector characterization and analysis was carried out by E.D. and J.P.H. with assistance from V.L., A.P.C., and A.R.J.M. E.D. and P.J.C. wrote the manuscript with input from all the coauthors.

\section{Funding}

Financial support for this work was provided by EPSRC (Grant No. EP/N018605/1), the Royal Academy of Engineering (Grant No. 10216/114), and the Joy Welch Educational Charitable Trust and the Lancaster University Research Committee.

\section{Notes}

The authors declare no competing financial interest.

\section{ACKNOWLEDGMENTS}

Financial support for this work was provided by EPSRC (Grant No. EP/N018605/1), the Royal Academy of Engineering (Grant No. 10216/114), and the Joy Welch Educational Charitable Trust and the Lancaster University Research Committee. The underlying data in this paper is available from https://dx.doi.org/10.17635/lancaster/researchdata/ 257. The authors would also like to acknowledge Prof. Eric Tournié and Dr. Jean-Baptiste Rodriguez of Institute of Electronics and Systems, University of Montpellier, for their helpful discussions.

\section{REFERENCES}

(1) Downs, C.; Vandervelde, T. Progress in Infrared Photodetectors since 2000. Sensors 2013, 13, 5054.

(2) Hoang, A. M.; Chen, G.; Chevallier, R.; Haddadi, A.; Razeghi, M. High performance photodiodes based on InAs/InAsSb type-II superlattices for very long wavelength infrared detection. Appl. Phys. Lett. 2014, 104, 251105.

(3) Lackner, D.; Steger, M.; Thewalt, M. L. W.; Pitts, O. J.; Cherng, Y. T.; Watkins, S. P.; Plis, E.; Krishna, S. InAs/InAsSb strain balanced superlattices for optical detectors: Material properties and energy band simulations. J. Appl. Phys. 2012, 111, 034507.

(4) Aytac, Y.; Olson, B. V.; Kim, J. K.; Shaner, E. A.; Hawkins, S. D.; Klem, J. F.; Olesberg, J.; Flatt, M. E.; Boggess, T. F. Bandgap and temperature dependence of Auger recombination in InAs/InAsSb type-II superlattices. J. Appl. Phys. 2016, 119, 215705.

(5) Olson, B. V.; Shaner, E. A.; Kim, J. K.; Klem, J. F.; Hawkins, S. D.; Murray, L. M.; Prineas, J. P.; Flatte, M. E.; Boggess, T. F. Timeresolved optical measurements of minority carrier recombination in a mid-wave infrared InAsSb alloy and InAs/InAsSb superlattice. Appl. Phys. Lett. 2012, 101, 092109.

(6) Haddadi, A.; Chevallier, R.; Chen, G.; Hoang, A. M.; Razeghi, M. Bias-selectable dual-band mid-/long-wavelength infrared photodetectors based on InAs/InAs ${ }_{1-\mathrm{x}} \mathrm{Sb}_{\mathrm{x}}$ type-II superlattices. Appl. Phys. Lett. 2015, 106, 011104.

(7) Maimon, S.; Wicks, G. W. nBn detector, an infrared detector with reduced dark current and higher operating temperature. Appl. Phys. Lett. 2006, 89, 151109.

(8) Klipstein, P. C. XBn barrier photodetectors for high sensitivity and high operating temperature infrared sensors. Proc. SPIE 2008, 6940, 69402U.

(9) Ting, D. Z.; Soibel, A.; Khoshakhlagh, A.; Sir Rafol, B.; Keo, S. A.; Hoglund, L.; Fisher, A. M.; Luong, E. M.; Gunapala, S. D. Midwavelength high operating temperature barrier infrared detector and focal plane array. Appl. Phys. Lett. 2018, 113, 021101.

(10) Prins, A. D.; Lewis, M. K.; Bushell, Z. L.; Sweeney, S. J.; Liu, S.; Zhang, Y.-H. Evidence of a defect level above the conduction band edge of InAs/InAsSb type-II superlattices for applications in efficient infrared photodetectors. Appl. Phys. Lett. 2015, 106, 171111.

(11) Lau, J. H. Recent advantages and new trends in flip chip technology. J. Electron. Packag. 2016, 138, 030802-1. 
(12) Thomson, D.; Zilkie, A.; Bower, J. B.; Komljenivic, T.; Reed, G. T.; Vivien, L.; Marris-Morini, D.; Cassan, E.; Virot, L.; Fédéli, J.-M.; Hartmann, J.-M.; Scmid, J. H.; Xu, D.; Boeuf, F.; O’Brien, P.; Mashanovich, G. Z.; Nedeljkovic, M. Roadmap on silicon photonics. J. Opt. 2016, 18, 073003.

(13) Akahane, K.; Yamamoto, N.; Gozu, S.; Ohtani, N. Heteroepitaxial growth of $\mathrm{GaSb}$ on $\mathrm{Si}$ (001) substrates. J. Cryst. Growth 2004, 264, 21-25.

(14) Matthews, J. W.; Blakeslee, A. E. Defects in epitaxial multilayers: I. Misfit Dislocations. J. Cryst. Growth 1974, 27, 118-125.

(15) Kim, Y. H.; Noh, Y. K.; Kim, M. D.; Oh, J. E.; Chung, K. S. Transmission electron microscopy study of initial growth stage of $\mathrm{GaSb}$ grown on $\mathrm{Si}$ (001) substrate by molecular beam epitaxy method. Thin Solid Films 2010, 518, 2280-2284.

(16) Ward, T.; Sánchez, M.; Tang, M.; Wu, J.; Liu, H.; Dunstan, D. J.; Beanland, R. Design rules for dislocation filters. J. Appl. Phys. 2014, 116, 063508.

(17) Haddadi, A.; Chen, G.; Chevallier, R.; Hoang, A. M.; Razeghi, M. InAs/InAs $s_{1-x} S b_{x}$ type-II superlattices for high performance long wavelength infrared detection. Appl. Phys. Lett. 2014, 105, 121104.

(18) Lackner, D. InAsSb/InAs strain balanced superlattices for photodetector applications. PhD Thesis, Simo Fraser University, 2011.

(19) Nguyen-Van, H.; Baranov, A. N.; Loghmari, Z.; Cerutti, L.; Rodriguez, J.-B.; Tournet, J.; Narcy, G.; Boissier, G.; Patriarche, G.; Bahriz, M.; Tournié, E.; Teissier, R. Quantum cascade lasers grown on silicon. Sci. Rep. 2018, 8, 7206.

(20) Wang, J.-B.; Ding, D.; Johnson, S. R.; Yu, S.-Q.; Zhang, Y.-H. Determination and improvement of spontaneous emission quantum efficiency in GaAs/AlGaAs heterostructures grown by molecular beam epitaxy. Phys. Status Solidi B 2007, 244 (8), 2740-2751.

(21) Burguete, C. G.; Guo, D.; Jurczak, P.; Cui, F.; Tang, M.; Chen, W.; Deng, Z.; Chen, Y.; Gutiérrez, M.; Chen, B.; Liu, H.; Wu, J. Direct growth of InAs/GaSb type II superlattice photodiodes on silicon substrates. IET Optoelectron. 2018, 12 (1), 2-4.

(22) Jia, B. W.; Tan, K. H.; Loke, W. K.; Wicaksono, S.; Yoon, S. F. Integration of an InSb photodetector on $\mathrm{Si}$ via heteroepitaxy for the mid-infrared wavelength region. Opt. Express 2018, 26 (6), 7227.

(23) Tennant, W. E. Rule 07 Revisited: Still a good heuristic predictor of $\mathrm{p} / \mathrm{n} \mathrm{HgCdTe} \mathrm{photodiode} \mathrm{performance?} \mathrm{J.} \mathrm{Electron.} \mathrm{Mater.}$ 2010, 39 (7), 1030-1035.

(24) Klipstein, P.; Klin, O.; Grossman, S.; Snapi, N.; Yaakobovitz, B.; Brumer, M.; Lukomsky, I.; Aronov, D.; Yassen, M.; Yofis, B.; Glozman, A.; Fishman, T.; Berkowitz, E.; Magen, O.; Shtrichman, I.; Weiss, E. MWIR InAsSb XBn detectors for high operating temperatures. Proc. SPIE 2010, 7660, 76602Y.

(25) Craig, A. P.; Marshall, A. R. J.; Tian, Z.-B.; Krishna, S.; Krier, A. Mid-Infrared InAsSb-based nBn photodetector with AlGaAsSb barrier layers, and comparison with InAsSb p-i-n diodes, both grown on GaAs using interfacial misfit arrays. Appl. Phys. Lett. 2013, 103, 253502.

(26) Michel, E.; Xu, J.; Kim, J. D.; Ferguson, I.; Razeghi, M. InSb infrared photodetector on $\mathrm{Si}$ substrates grown by molecular beam epitaxy. IEEE Photonics Technol. Lett. 1996, 8, 673.

(27) Jia, B. W.; Tan, K. H.; Loke, W. K.; Wicaksono, S.; Lee, K. H.; Yoon, S. F. Monolithic integration of InSb photodetector on silicon for mid-infrared silicon photonics. ACS Photonics 2018, 5, 15121520.

(28) Korkmaz, M.; Arikan, B.; Suyolcu, E.; Aslan, B.; Serincan, U. Performance evaluation of InAs/GaSb superlattice photodetector grown on GaAs substrate using AlSb interfacial misfit array. Semicond. Sci. Technol. 2018, 33, 035002. 